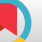

\title{
Pregnant Women's Knowledge and Beliefs About Voluntary HIV Counseling and Testing in Birjand in 2018: An Assessment Using Health Belief Model
}

\author{
Gholamreza Sharifzadeh (iD ${ }^{1}$, Mohammad Ali Behdani (iD ${ }^{2,}{ }^{*}$ and Mitra Moodi (iD ${ }^{3}$ \\ ${ }^{1}$ Social Determinants of Health Research Center, Faculty of Health, Birjand University of Medical Sciences, Birjand, Iran \\ ${ }^{2}$ Student Resarch Committee, Birjand University of Medical sciences, Birjand, Iran \\ ${ }^{3}$ Department of Health Education and Health Promotion, Social Determinants of Health Research Center, Faculty of Health, Birjand University of Medical Sciences, Birjand, \\ Iran \\ "Corresponding author: Student Resarch Committee, Birjand University of Medical sciences, Birjand, Iran. Email: alibehdani@bums.ac.ir
}

Received 2019 December 09; Revised 2020 September 06; Accepted 2020 September 09.

\begin{abstract}
Background: Pregnant women are at increased risk of HIV acquisition, whose rapid diagnosis not only saves the lives of the mother and fetus and prevents the infection of the husband, but also prevents the transmission of the virus to other people in the community.

Objectives: In this line, this study aimed to assess pregnant women's knowledge and beliefs in Birjand about voluntary HIV counseling and testing based on health belief model constructs.

Methods: In this descriptive-analytical study, 238 pregnant women referring to Birjand comprehensive health centers (CHC) in 2018 are studied. Participants were selected using multi-stage random sampling. First, Birjand was divided into four districts as north, south, east, and west. Then, one health center and one CHC were selected from each district. Data were collected using a researchermade questionnaire consisting of demographic information, knowledge, model constructs, and behavior, whose validity and reliability were determined. Data were analyzed using SPSS19 and independent t-test and one-way ANOVA.

Results: The mean age of participants was $28.3+5.1$ years. Mean score of knowledge, susceptibility, severity, benefits, barriers and perceived self-efficacy were $5.9+3.6,8.9+2.21,23.7+4.7,34.7+4.2,28.2+5.9$, and $24.5+7.3$ respectively and behavior was determined to be $5 \%$. Knowledge was evaluated to be poor in $1.7 \%$, moderate in $51.7 \%$, and good in $46.6 \%$. There was a significant negative relationship between barriers and all model constructs $(\mathrm{P}<0.05)$ and a significant positive relationship between knowledge with perceived susceptibility $(r=0.15)$ and guidance for action $(r=0.2)$, susceptibility with perceived severity $(r=0.23)$.

Conclusions: This study showed the insufficiency of pregnant women's knowledge about the importance of HIV screening. Therefore, it is recommended to use the Health Belief model in $\mathrm{CHC}$ to improve the knowledge, attitude, and practice of this target group.
\end{abstract}

Keywords: HIV, Pregnant Women, Health Belief Model, Knowledge

\section{Background}

Nowadays, HIV, a virus that causes AIDS, is one of the major public health challenges worldwide (1). Iran is at a critical stage of the AIDS epidemic, and the country is facing the third wave of the disease, mainly through unsafe sexual intercourse (2).

So far, more than 32 million people have died of AIDS, and in 2018 nearly 1.3 million pregnant women were infected with HIV (3). According to the World Health Organization report in 2014, about 1.5 million pregnant women had AIDS at that time. The feminization of the HIV/AIDS epidemic is directly associated with an increased number of infected children through vertical transmission of the dis- ease (2).

Since the prevalence of HIV/AIDS is on the rise among Iranian women, who most of them are at fertile age (2), special attention should be paid to the mother-to-child (MCTC) transmission and its effective prevention methods as well as ethical considerations. Given the limitations of HIV screening-related resources, we recommend offering HIV testing to every pregnant woman at the first clinic visit by providing enough information and respecting their willingness (4). The most important obstacle to effective prevention of HIV/AIDS is the insufficiency of knowledge about different aspects of the disease. Therefore, understanding the level of knowledge of various target groups can be considered as the first step in designing educa- 
tional interventions (5). Several studies have reported that knowledge about HIV/AIDS, and voluntary counseling and testing are key strategies in managing and preventing HIV (6). A number of models are proposed for behavior change, which one of the effective models specially developed for health education is the Health Belief model (HBM). In this model, behavior is a function of perceived knowledge and attitude. Based on the constructs of the HBM, it predicts behaviors by accounting health threats and driving the behaviors toward health. Therefore, for adopting AIDS prevention behaviors (primary prevention), people first have to feel threatened (perceived susceptibility), then understand the depth of risk and the seriousness of its various side effects (perceived severity), with the positive symptoms they receive from their surroundings (cues to action) they will believe the usefulness and applicability of preventive behaviors (perceived benefits), finding should find factors that prevent these behaviors are also less costly than their benefits (perceived barriers) and also should be able to perform preventive behaviors (perceived selfefficacy) until so that they finally, to achieve the proper performance in the field of AIDS prevention (7). So that they finally take the appropriate measures in preventing AIDS.

\section{Objectives}

Based on the aforementioned, pregnant women are at increased risk of HIV acquisition, whose rapid diagnosis not only saves the lives of the mother and fetus and prevents the infection of the husband but also prevents the transmission of the virus to other people in the community. In this line, the present study aimed to assess pregnant women's knowledge and beliefs in Birjand about voluntary HIV counseling and testing based on health belief model constructs.

\section{Methods}

In this cross-sectional descriptive-analytic study, 238 pregnant women referring to comprehensive health centers (CHC) of Birjand in 2018 are studied. Those who were not willing to participate or did not complete the questionnaire completely were excluded from the study. Participants were selected using multi-stage random sampling. First, Birjand was divided into four districts (north, south, east, and west). Then, one health center and one $\mathrm{CHC}$ were selected from each district. Afterward, 50 pregnant women referring to midwives to receive consultation were selected from each district by easy non-probability sampling method. Then, participants were asked to fill the questionnaire. The sample size was calculated as 238 , based on the purpose of determining the frequency distribution of voluntary HIV testing, ratio estimation formula, and the methodology followed by Almo et al. (8) study $(\propto=0.05 ; \mathrm{P}$ $=0.67 ; \mathrm{d}=0.06$ ).

Data were collected using a researcher-made questionnaire, which was developed based on the literature review and health belief model constructs. It consists of demographic items (age, delivery mode, number of children, education of the pregnant women and her husband, history of drug injections, tattoos, ... (13 questions)), knowledge items (with correct, false, and do not know choices (15 questions)), perceived susceptibility and severity items (11 questions), perceived benefits ( 9 questions), perceived barriers (10 questions), self-efficacy (7 questions), and cues to action (5 questions).

Three-choice questions (yes, no, and don't know) were used to assess the knowledge of participants, which for correct answer a score of 2, for false answer a score of zero, and don't know a score of one was considered. A five-point Likert scale (completely agree to completely disagree) was used to measure the health belief model's constructs. In the construct of self-efficacy, five options were used (not sure, a little confident, moderately confident, very confident, completely confident), the scores of these answers ranged from one to five, respectively.

Scores generally ranged from 0 to 30 . In perceived susceptibility from 3 to 15 , in perceived severity from 8 to 40 , in perceived benefits from 9 to 45 , in perceived barriers from 10 to 50 , and in self-efficacy from 7 to 35 . Scores less than $50 \%$ of the total score were considered as weak, between 50 to $75 \%$ of the total as moderate, and above $75 \%$ of the total score were classified as a good level.

Using opinions of 10 university professors of the School of Public Health of the Birjand University of Medical Sciences, the validity of the questionnaire was assessed (CVI $=0.97$ and $\mathrm{CVR}=0.86$ ). To assess the reliability of the construct questions, the questionnaire was completed by 29 pregnant women referring to CHCs. For each construct, the Cronbach's alpha was as follows: perceived susceptibility 0.71 , perceived severity 0.72 , perceived benefits 0.60 , perceived barriers 0.65 , self-efficacy 0.91 , and total constructs 0.73 .

Before completing the questionnaire, the required information on how to respond to the questions were provided to participants. The questionnaire was completed as a self-report.

The data were entered into SPSS version 19 and were analyzed using descriptive statistics. First, the normality of the distribution of data was evaluated. Independent $t$-test, one-way ANOVA, and Tukey post hoc tests were used to analyze the data. A P value of $<0.05$ was considered statistically significant. 


\section{Results}

In this study, 238 pregnant women with a mean age of $28.3 \pm 5.1$ years were studied. The youngest and oldest participants were 16 and 42 years, respectively. Of 238 pregnant women, 20 ( $8 \%$ ) had primary and guidance education degrees, 89 (37\%) had a high-school degree, and 129 (54\%) had a university degree. Also, 186 (78\%) were housewives, and the rest were employed. The mean score of each construct was as follow knowledge $5.9 \pm 3.6$, perceived susceptibility $8.9 \pm 2.21$, perceived severity $23.7 \pm 4.7$, perceived benefits $34.7 \pm 4.2$, perceived barriers 28. $2 \pm 5.9$, selfefficacy $24.5 \pm 7.3$, and guideline for operation $1.8 \pm 1.3$.

The mean score of knowledge and perceived susceptibility was directly associated with a higher level of education. While for perceived barriers, this association was indirect. According to the results of the Turkey test, the difference between university degrees and the other three levels was significant $(\mathrm{P}<0.001)$. Also, for the perceived barriers construct, the Tukey test showed a significant difference between the elementary degree and other degrees (Table 1).

As shown in Table 2, the mean score of knowledge 2 was significantly higher in employed women than in housewives $(\mathrm{P}=0.008)$.

The association between model structures is presented in Table 3, which indicates a significant negative association between barriers and all model structures $(\mathrm{P}<0.05)$ and a significant positive association between knowledge with perceived susceptibility, cues to action, sensitivity, and perceived severity.

Knowledge in $46.6 \%$ of cases, perceived sensitivity $14.3 \%$, perceived severity $6.7 \%$, perceived benefits $49.6 \%$, perceived barriers $25.2 \%$, and self-efficacy in $45.8 \%$ were categorized as good (Table 4 ).

Concerning the cues to action, the most popular channel (used by 150 subjects or 63\%) to obtain the required information about HIV was media, followed by 94 (39\%) through announcements and posters at $\mathrm{CHCs}$ or physical advertisements.

\section{Discussion}

This study showed that $47 \%$ of pregnant women had good knowledge about HIV, and there was a correlation between knowledge and perceived susceptibility constructs. This means that targeted efforts to raise people's knowledge as a moderating factor can improve scores on HBM constructs and, eventually, leads to improved behavior.

Also, the relatively lower level of housewife women's awareness about HIV compared to their employed counterparts indicates the necessity of focusing educational programs on this group. The results reported by Asaduzzaman et al. (9), Olwoker et al. (10), Heshmati et al. (11) about the knowledge of housewife women are consistent with this study. Since perceived susceptibility is the most important predictor of behavior (12), and in this study, $14 \%$ of pregnant women had good perceived sensitivity (55\% were moderate), this finding indicates the susceptibility of this group to HIV infection. On the other hand, mothers' low knowledge has reduced their sensitivity. Studies by KhaniJeihouni et al. (13) and Heshmati et al. (11) are in agreement with the above findings, but in the study by Pirzadeh et al. (14) participants were highly sensitive.

Despite low perceived susceptibility in mothers under study, perceived severity was moderate for $73 \%$ of the participants, which shows their understanding of the seriousness of the disease and the irreversible consequences of AIDS. While this is notable, it also indicates that mothers do not consider themselves as much at risk as they consider the disease to have severe consequences.

However, since pregnant women who participated in the current study were not considering themselves at increased risk of HIV acquisition, the finding seems to be realistic. This result is consistent with the study of Heshmati et al. (11), but inconsistent with Pirzadeh et al. (14).

For most pregnant women, the perceived barrier to HIV testing was about $70 \%$ and the mean score of the construct was not satisfying. This indicates that people cannot overcome these barriers. It also reveals the social stigma towards HIV-positive individuals and low knowledge about the disease, that was mentioned earlier. Which in turn creates barriers for preventive behaviors, including HIV testing. And indicates the need for increasing the awareness of society to remove or reduce these barriers. The results of this study were in line with the results of Mahdavifar et al. (15) and Khani-Jeihouni et al. (13).

Self-efficacy is the principle that links one's knowledge, behavior, and beliefs about behavior. Not only awareness about the causes of behavior is necessary, but also we should be able to do that behavior. In the current study, the mean score of self-efficacy was somehow desirable, which is in line with the findings of Mahdavifar's (15). Voluntary HIV testing was positively associated with educational attainment, which is consistent with the study of Alamo et al. (8) and Matenga et al. (16). In this study, 5\% of women were willing to perform the HIV screening test. Montazeri argued that even when the benefits are well-perceived, behavior change is still difficult as long as the perceived barriers are not removed (15).

\subsection{Conclusions}

The only method to curb the HIV epidemic and meeting goals announced by the WHO is the elimination of social stigma towards HIV, which subsequently promotes using screening tests and receiving treatment. Since the 


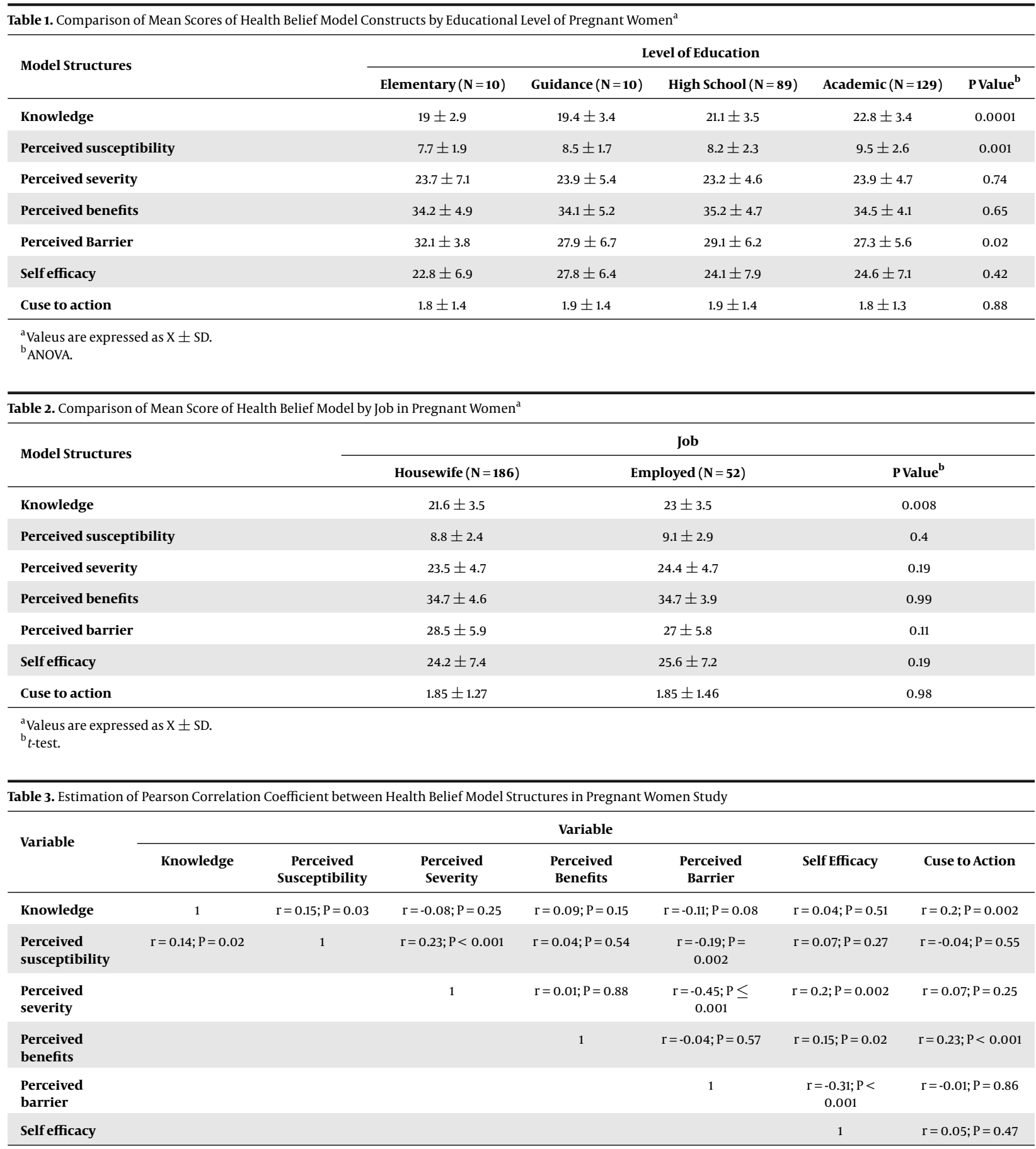

most effective way against HIV is health education and without increasing the knowledge and self-efficacy and decreasing barriers that pregnant women are faced with, it is impossible to increase the utilization of HIV screening tests. Thus, based on the results, it is recommended to use the HBM in $\mathrm{CHC}$ to improve knowledge, attitude, and prac- tice of pregnant women.

\section{Acknowledgments}

The authors would like to thank the Vice-Chancellor for Research Affairs of the Birjand University of Medical Sci- 


\begin{tabular}{|c|c|c|c|c|}
\hline \multirow{2}{*}{ Variable } & \multicolumn{4}{|c|}{ Status } \\
\hline & Poor & Medium & Good & Total \\
\hline Knowledge & $4(1.7)$ & $123(51.7)$ & $111(46.6)$ & $238(100)$ \\
\hline Perceived susceptibility & $72(30.3)$ & $132(55.5)$ & $34(14.3)$ & $238(100)$ \\
\hline Perceived severity & $46(19.3)$ & $176(73.5)$ & $16(6.7)$ & $238(100)$ \\
\hline Perceived benefits & $1(0.4)$ & $119(50)$ & $118(49.6)$ & $238(100)$ \\
\hline Perceived barrier & $13(5.5)$ & $165(69.3)$ & $60(25.2)$ & $238(100)$ \\
\hline self-efficacy & $54(18.6)$ & $84(35.3)$ & $106(45.8)$ & $238(100)$ \\
\hline
\end{tabular}

${ }^{\mathrm{a}}$ Values are expressed as No. (\%).

ence for their support and to the pregnant women who participated in the current study.

\section{Footnotes}

Authors' Contribution: The authors equally contributed to the writing and revision of this paper.

Conflict of Interests: The authors declare no conflict interest.

Ethical Approval: The current study is confirmed by the Ethics Committee of the Birjand University of Medical Sciences (code: IR.BUMS.REC.1397.140).

Funding/Support: None declared.

\section{References}

1. WHO. HIV/AIDS. 2020, [cited 2020 Jul 24]. Available from: https://www. who.int/news-room/facts-in-pictures/detail/hiv-aids.

2. Bahri N, Bajdi A, Latifnejad Roodsari R, Mirzaee K, Esmaeili H, Larki M. Perceived and real educational needs of midwives about prevention of Mother-to-Child Transmission of HIV,(Hospitals affiliated to Mashhad University of Medical Sciences-2015). Iran J Obstet Gynecol Infertil. 2016;19(21):12-21.

3. WHO. HIV/AIDS Media centre. 2020, [cited 2020 Jul 06]. Available from: https://www.who.int/en/news-room/fact-sheets/detail/hiv-aids.

4. Salari P, Azizi M. The necessity of HIV testing in Iranian pregnant women and its ethical considerations. J Med Ethics Hist Med. 2009;2.

5. Auerbach JD, Coates TJ. HIV prevention research: accomplishments and challenges for the third decade of AIDS. Am J Public Health. 2000;90(7):1029-32. doi: 10.2105/ajph.90.7.1029. [PubMed: 10897177]. [PubMed Central: PMC1446304].

6. Buldeo P, Gilbert L. Exploring the Health Belief Model and first-year students' responses to HIV/AIDS and VCT at a South African university. Afr J AIDS Res. 2015;14(3):209-18. doi: 10.2989/16085906.2015.1052527. [PubMed: 26284910].
7. Glanz K, Rimer BK, Viswanath K. Health behavior and health education: theory, research, and practice. John Wiley \& Sons; 2008.

8. Alemu YM, Ambaw F, Wilder-Smith A. Utilization of HIV testing services among pregnant mothers in low income primary care settings in northern Ethiopia: a cross sectional study. BMC Pregnancy Childbirth. 2017;17(1):199. doi:10.1186/s12884-017-1389-2. [PubMed: 28646888]. [PubMed Central: PMC5483315].

9. Asaduzzaman M, Higuchi M, Sarker MA, Hamajima N. Awareness and knowledge of HIV/AIDS among married women in rural Bangladesh and exposure to media: a secondary data analysis of the 2011 Bangladesh Demographic and Health Survey. Nagoya J Med Sci. 2016;78(1):109-18. [PubMed: 27019532]. [PubMed Central: PMC4767519].

10. Olowokere AE, Adelakun OA, Komolafe AO. Knowledge, perception, access and utilisation of HIV counselling and testing among pregnant women in rural communities of Osogbo town, Nigeria. Aust JRural Health. 2018;26(1):33-41. doi: 10.1111/ajr.12368. [PubMed: 29239073].

11. Heshmati H, Behnampour N, Rezaei B, Pirzadeh A. A study on highschool students' knowledge about the aids in Ashkhane city, Iran, 2012, applying Health Belief Model. Health Serv Res. 2016;12(1):1-5.

12. Carmel S. The Health Belief Model in the research of AIDS-related preventive behavior. Public Health Rev. 1990;18(1):73-85.

13. Khani-Jeihouni A, Ranjbari S, Khiyali Z, Moradi Z, Motamedi MJ. Evaluation of the Factors Associated with AIDS Prevention Performance among Male Barbers Based on the Health Belief Model in Fasa. JECH. 2017;3(4):59-65. eng. doi:10.21859/jech.3.4.59.

14. Pirzadeh A, Sharifirad GHR. Effect of educational program on knowledge and health belief model structures about acquired immune deficiency syndrome (AIDS) among high school female student in Isfahan, Iran.J Gorgan Univ Med Sci. 2012;14(3):66-71.

15. Mahdavifar M, Sabzevari S, Fasihi HT. Using health belief model for assessing health believes about papsmear in women referred to health care centers in Bandar Abbas, Iran. J Health Dev. 2015.

16. Mtenga SM, Exavery A, Kakoko D, Geubbels E. Social cognitive determinants of HIV voluntary counselling and testing uptake among married individuals in Dar es Salaam Tanzania: Theory of Planned Behaviour (TPB). BMC Public Health. 2015;15:213. doi: 10.1186/s12889-0151545-4. [PubMed: 25885001]. [PubMed Central: PMC4355471]. 